\title{
ACOUSTIC INVESTIGATION OF THE STRUCTURE OF MAGNETO-RHEOLOGICAL FLUID
}

The acoustic spectroscopy is used to study properties and changes in structural arrangement in silicone oil based magneto-rheological fluids with carbonyl iron particles upon the effect of an external magnetic field. Attenuation spectra at three temperatures for various concentrations of magnetic particles are presented. The attenuation of acoustic waves was measured for a jump change of the magnetic field to $200 \mathrm{mT}$ as a function of the temperature. The relaxation effects for the acoustic attenuation after switching off the magnetic field and its decrease to the similar value as for clean silicone oil were observed. The change of acoustic attenuation in magneto-rheological fluid versus angle between the wave vector of acoustic waves and direction of the applied magnetic field was measured, too. For the anisotropy measurement are characteristic two local maxima from which results chain orientation in direction of the magnetic field.

Keywords: Particle structure, magneto-rheological fluid, acoustic spectroscopy, anisotropy.

\section{Introduction}

Magneto-rheological (MR) fluids are defined as suspensions of magnetic particles in a carrier liquid with some additives to stabilize this smart system. The particles are of sizes between 1 to 10 microns. In the absence of an external magnetic field, MR fluids are reasonably well approximated as Newtonian liquids. A problem of these systems is the sedimentation due to the density difference between microparticles and carrier liquid. Surfactants are often added to delay the sedimentation process and re-disperse the particles easily [1, 2]. Their rheological properties (e.g. viscosity) are rapidly varied by applying the external magnetic field. Changes in their properties are caused by efficiency of magnetic microparticles polarization, affected further by shear conditions, magnetic field expressed herein in terms of magnetic flux density, $\boldsymbol{B}$, and its direction, structural and physicochemical characteristics of dispersed phase, volume concentration of magnetic particles, carrier liquid properties, stabilization components, as it was founded for the similar electrorheological fluids [3, 4]. When the external magnetic field is applied, a magnetic dipole moment is induced in the particles parallel to the magnetic field and as the result; particles tend to align forming ordered structures, giving rise to significant variations not only in viscosity but also in its inner order (particle rearrangement). In most engineering applications a simple Bingham plastic model is effective for describing the field- dependent fluid characteristics. So they can be applied in various fields of civil engineering, safety engineering, transportation and life science, in the design of brakes, dampers, clutches and shock absorbing systems [5 - 7].

The corrosion, oxidation, and abrasive properties of iron and iron alloys frequently used as optimal magnetic agents in MR fluids are, however, obstacles for their wider commercial usage. The modification of particle surface by chemical process has proven to be efficient to overcome this problem [6, 8]. The improvement of properties of MR fluids by plasma-enhanced chemical vapour deposition of octafluorocyclobutane onto carbonyl iron (CI) particles using rotary plasma reactor was used in work [8]. The results revealed successful fluorination of CI particles with a maximum of fluorine content of $2.9 \%$. The fluoropolymer film fabricated onto particles improved their corrosion protection and friction properties by $40 \%$. Amorphous alloy particles can be used instead of the traditional CI particles as the dispersed phase to prepare amorphous based MR fluid [Dong6]. The amorphous particles have larger magnetization intensity and permeability at lower field intensities as well as lower density. MR performance and sedimentation stability are better in the MR fluid based on amorphous particles than that based on conventionally used CI particles. Magneto-rheological fluids exhibit some advantages over typical electrorheological (ER) materials. In contrast to ER materials, MR fluids are more useful because the change in their rheological properties is more pronounced compared to ER fluids,

\footnotetext{
* ${ }^{1}$ Jozef Kudelcik, ${ }^{1}$ Peter Bury, ${ }^{1}$ Stefan Hardon, ${ }^{2}$ Michal Sedlacik, ${ }^{2}$ Tomas Plachy

${ }^{1}$ Department of Physics, University of Zilina, Slovakia

${ }^{2}$ Centre of Polymer Systems, University Institute, Tomas Bata University in Zlin, Czech Republic

E-mail: kudelcik@fyzika.uniza.sk
} 
i.e. an increase of yield stress is 20-55 times higher [5]. There are also electro-magneto-rheological fluids, which consist of particles responsive to both electric and magnetic fields. The results from the molecular dynamic simulation studies have shown that under these fields various types of structural formation and transition between them can exist [9].

The fluids with microstructure and particle aggregation are studied using optical measurements $[1,10]$, the dielectric spectroscopy [11 - 14] and also there are a few works using ultrasonic techniques $[15,16]$. These ultrasonic techniques are appearing advantageous when the number of particles is high and optical observation becomes difficult. Some aspects of the acoustic behavior between magnetic fluids (MF) and MR fluids were compared and in other work properties such as the anisotropic effect and hysteresis when a uniform magnetic field is applied were studied. It should be noted that the theories describing action of magnetic field on mechanism of acoustic wave interaction with structure in colloidal fluids are not well developed and there are not experimental data reliable enough on this problem. The former theories were created only for diluted dispersive mediums and did not take into account very important processes of the internal ordered structures from magnetic particles formation in the fluid, volume and interaction of particles and its aggregates at the boundary. Currently, there are only few papers involved in the acoustic properties of MR fluids as studied in this work. One of them is work by Baev, et all. [3], who made similar acoustic measurement for higher concentration of magnetic particles, but with different type of MR fluid. Data analysis confirmed dependence of the sound velocity and the acoustic attenuation on the magnitude and direction of magnetic field in MR fluid. Their measurement also showed that interparticle interactions also depend on the processes in the interface boundary.

Many other works are focused on the structural changes in MF with magnetic nanoparticles by the influence of the magnetic field using the acoustic spectroscopy [3, 7, 17 - 20]. The diameters and magnetic moments of magnetic nanoparticles in stable colloidal solutions are much lower than in MR fluids. Under the effect of the magnetic field, the nanoparticles within MF become arranged into oligomers, chains or clusters. The propagation of the acoustic wave in MF and change of acoustic attenuation by magnetic field was studied by several authors both theoretically and experimentally [20 - 22].

\section{Experiment}

Carbonyl iron particles (HQ grade, BASF, Germany) were used for preparation of MR fluid, which was investigated by acoustic methods. The main material characteristics of CI particles with $\mathrm{HQ}$ grade according to the manufacturer are following: spherical shape of particles with the average size of about $1 \mu \mathrm{m}$, non-modified surface, and content of $\alpha$-iron $>97 \%$. Carbonyl iron particles are soft magnetic particles since their increasing and decreasing branches of the hysteresis cycles are hardly distinguishable, the magnetization saturation in the field of $777 \mathrm{kA} \cdot \mathrm{m}^{-1}$ is $174 \mathrm{emu} \cdot \mathrm{g}^{-1}$. The MR fluids with the particle concentration of 1 and $2 \mathrm{wt} . \%$ in silicone oil - Lukosiol M50 [23] were prepared. Figure 1 shows the size and surface morphology of CI particles employed as a dispersed phase in MR fluid under investigation. All the particles are evidently of a spherical shape with the average size of about $1 \mu \mathrm{m}$ confirming the manufacturer data.

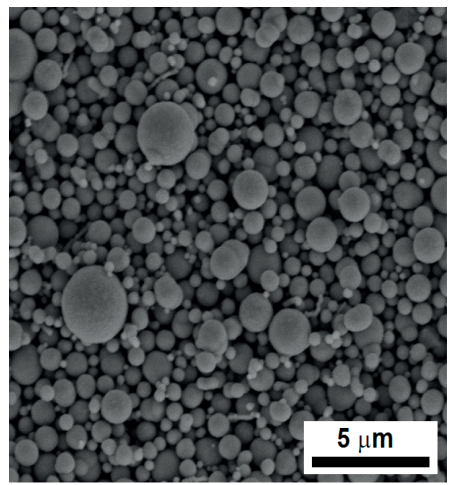

Fig. 1 SEM image of CI particles under investigation

The ultrasound spectrometer DT-100, as the first acoustic method, was used for the characterization of MR fluids (Fig. 2). In addition to the acoustic sensor, probes to measure the temperature and $\mathrm{pH}$ are incorporated. The controller box sent a high frequency signal, which was transferred from the deepseated sender into an ultrasound wave by means of a piezo transducer. The frequency range of the DT-100 is $1-100 \mathrm{MHz}$ and the gap between sound-sender and - detector is variable from 0.1 to $21 \mathrm{~mm}$. The wave was attenuated in the MR fluid and the signal was detected by the receiver at the opposite ultrasound transducer and used for the particle size analysis. The principle of measurement is the so called „tone-burst"-method: short wave packets with a very narrow frequency distribution and a few numbers of oscillation cycles are sent through the dispersion chamber (Fig. 2) filled with the MR fluid. Sound attenuation and - speed are measured by detecting signal attenuation and - delay: The program using experimental data and integrated modern theories allows the determination of particle sizes in the range of $5 \mathrm{~nm}$ to $1000 \mu \mathrm{m}$ at a concentration range of 1 to $50 \mathrm{vol. \%}$ [24].

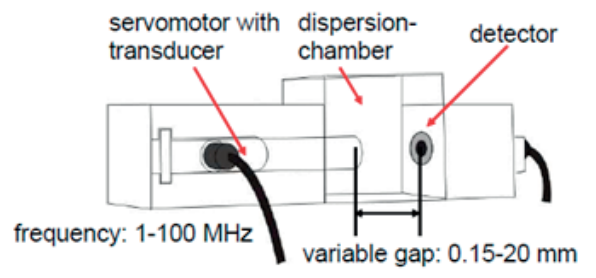

Fig. 2 The principal scheme of ultrasound spectrometer DT 100 [25] 
An ultrasound wave, which is transmitting through a liquid dispersion, is attenuated with the increasing path length. Basically, there are three effects responsible for this: intrinsic absorption, dissipative absorption (visco-inertial, thermal and structural effects) and sound scattering. After measuring the acoustic attenuation spectrum, the theoretical fit-spectrum is automatically adapted to the measured spectrum. The particle size distribution is based on the calculated best fit-parameters. The used model for the calculation or the combination of the models depends on the type of the dispersed phase(s). In our case for fine particles $(<5$ $\mu \mathrm{m})$ just the dissipative mechanism is important. In case of rigid particles (e.g. ceramics, metals), only the visco-inertial effect will be considered [25].
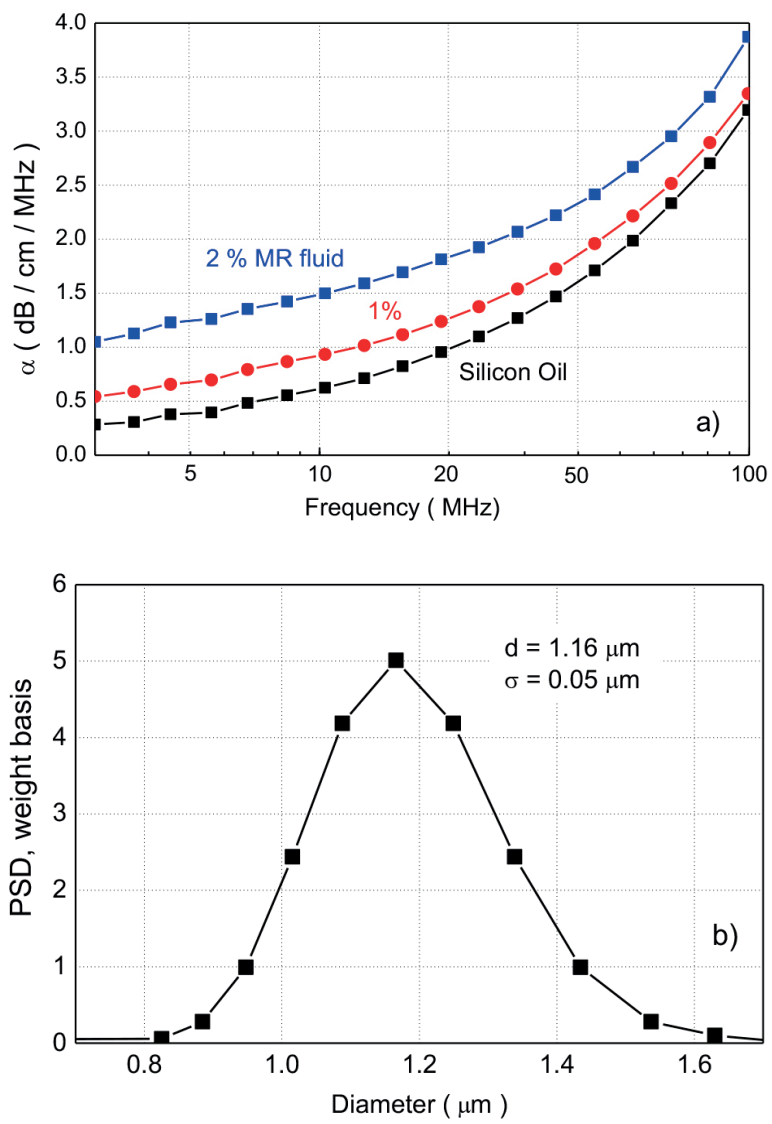

Fig. 3 a) Attenuation spectra measured for MR fluids and silicone oil; b) Size distribution of $2 \% \mathrm{wt}$. MR fluid at $20^{\circ} \mathrm{C}$ ( $d$-mean diameter of magnetic nanoparticles, $\sigma$-standard deviation)

Two different concentrations ( 1 and 2 wt.\%) of MR fluid based on silicone oil LUKOSIOL M50 with microscale particles carbonyl iron of HQ grade were measured. The samples were analysed regarding their attenuation spectra with stirring with external motor. All measurements were repeated twice in order to test the stability of the sample in the limits of the measurement period. The measured attenuation spectra are given in Fig. 3a). Beyond the intrinsic attenuation spectrum of pure silicon oil is shown. All dispersions are stable in the limits of the measurement period. The measurements were also made for various temperatures. Attenuation of all samples slightly decreased with increasing temperature. The main attenuation effect, which plays a role here due to the small size of the particles, is the viscous effect. The attenuation for MR fluid is higher because micro-particles cause additional dissipative attenuation by structural effect. The particle size distribution (PSD) for 2 wt.\% MR fluid is presented in Fig. $3 b)$. The diameter of carbonyl iron particles calculated from measured distribution agrees with the manufacturer data.

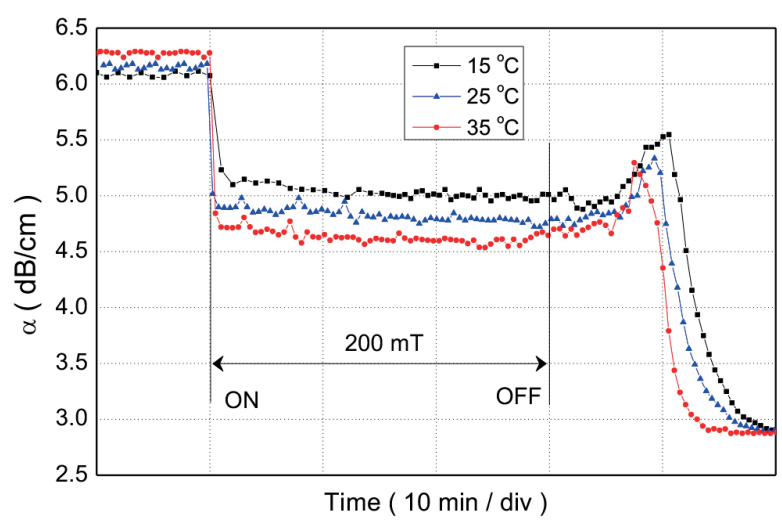

Fig. 4 Experimental data of the changes of acoustic attenuation for a jump change of the magnetic field to $200 \mathrm{mT}$ at various temperatures for 2 wt.\% MR fluid

The second acoustic method was used for the study of structural changes occurring during the application of external magnetic field. The experimental equipment used for this type of measurement was the same as in our previous works [26, 27]. The frequency of the ultrasonic wave was $4.9 \mathrm{MHz}$ with pulse length $0.6 \mu \mathrm{s}$. The changes in the ultrasonic wave attenuation were measured by the pulse method based on measurement of intensity of the ultrasonic pulse passed through or reflected by the medium studied. The MR fluid was placed in the thermostated closed measuring cell $\left(1.5 \times 0.9 \times 1 \mathrm{~cm}^{3}\right.$, temperature stabilization with an accuracy $\pm 0.1{ }^{\circ} \mathrm{C}$ by JULABO Refrigerated \& Heating Circulators $\mathrm{F} 25-\mathrm{HE}$ ) and the distance between two piezoelectric transducers was $9 \mathrm{~mm}$.

Figure 4 presents the changes of acoustic attenuation for a jump change of the magnetic field to $200 \mathrm{mT}$ for $2 \mathrm{wt} . \% \mathrm{MR}$ fluid at $15{ }^{\circ} \mathrm{C}, 25^{\circ} \mathrm{C}$, and $35{ }^{\circ} \mathrm{C}$, respectively. The given value of magnetic field was kept constant during next $30 \mathrm{~min}$. As can be seen from the measured development, the change of acoustic attenuation follows immediately the step change of the magnetic field and after a few minutes the acoustic attenuation was stable. The developments are almost the same, only with the increase of the temperature smaller stabilized values can be observed. After the magnetic field was switched off, the value of acoustic attenuation did not change during next $5 \mathrm{~min}$. After this time 
the acoustic attenuation increased and then it exponentially decreased with time constant of about $500 \mathrm{~s}$. The value of acoustic attenuation decreased during next minutes to the value as was measured for clean silicone oil.

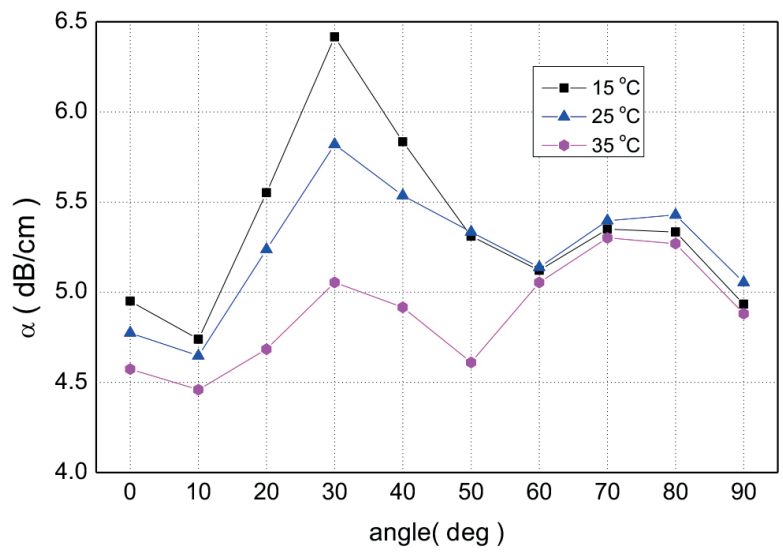

Fig. 5 Anisotropy measurement of the acoustic attenuation at constant magnetic field of $200 \mathrm{mT}$ for $2 \mathrm{wt} . \%$ MR fluid at various temperatures

Figure 5 illustrates the anisotropy of acoustic attenuation within the 2 wt.\% MR fluid at constant value of magnetic field applied $(200 \mathrm{mT})$ studied in the dependence on the angle $\phi$ between wave vector $\boldsymbol{k}$ and the magnetic field $\boldsymbol{B}$ measured for three different temperatures. The results indicate an effect of the angle on the acoustic attenuation within the MR fluid. The development of the anisotropy depends on the temperature, but for all cases it has similar characteristics. The acoustic attenuation firstly slowly decreases and then it increases to the angle $30^{\circ}$, where is the first local maximum. With the next increase of the angle attenuation decreases and it again increases to the second local maxima at the angle around $75^{\circ}$. The value of this local maximum is independent of the temperature. Finally, in perpendicular orientation of the magnetic field and the wave vector of acoustic wave, the acoustic attenuation decreases to the value which is slightly higher than for parallel orientation.

Interesting phenomena was observed when the magnetic field was switched off after anisotropy measurement. The time of the application of magnetic field during this type of measurement was more than $150 \mathrm{~min}$. In this case for each temperature the immediate decrease of acoustic attenuation to value as for clean silicone oil was observed. There was no relaxation time as is observed for the results present in Fig. 4 when the magnetic field was applied only $30 \mathrm{~min}$.

\section{Discussion}

The study of MR fluids by the acoustic spectroscopy provides important information about their properties and structures. The mean diameter determined by acoustic spectrometer DT-100 agrees with manufacture data quite well. The acoustic attenuation at frequency $4.9 \mathrm{MHz}$ is the same as from the second method used for study of structural changes of MR fluid in the presence of magnetic field. Thus, the acoustic spectroscopy is a very useful method for studying the structure changes occurring in MR fluids, which are the result of the interaction between the magnetic field applied and the magnetic moment of the microparticles generated by this field. These structures enlarge with the magnetic field and this process has the influence on the value of the acoustic attenuation. Similar results were observed also in works [17, 20 22] for MF with nanoparticles.

Magneto-rheological fluids contain microparticles which have chaotic distribution in fluid as depicted in Fig. 6a. In the presence of an external magnetic field, the particles acquire a dipole moment aligned with the field causing the particles can form linear chains aligned in direction with the external magnetic field. (Fig. 6b). This arrangement is confirmed by other measurements $[5,7,16]$ where the degree of change is related to the magnitude of the applied magnetic field, and can occur in a time scale of a few milliseconds. The similar quick effect on the acoustic attenuation after the application of magnetic field was observed (Fig. 4). After the immediate change of the acoustic attenuation, the next development depends on the magnitude of applied field and concentration. In the case of 2 wt.\% MR fluid for $100 \mathrm{mT}$ and higher values the stable state or the constant value of acoustic attenuation during the whole time of application of magnetic field was observed [27]. This stable arrangement of microparticles is the result of higher numbers of particles, thus produce stable chains. From the realized experiment it can be seen that for all temperatures stable value of acoustic attenuation was observed.

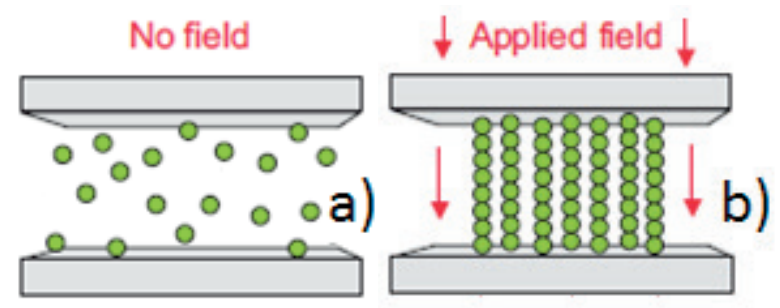

Fig. 6 MR fluid model without (a) and under

(b) the magnetic field applied

After switching off the magnetic field, a few interesting effects were observed. The first of them is that no significant changes in the relative long time lasting acoustic attenuation were noted during $5 \mathrm{~min}$. This effect could be described by a long lifetime, times of chains and an interaction of dispersive phase on the boundary fluid-transducer. The second effect is suddenly increased of the acoustic attenuation after switching the magnetic field off. This can be the result of slowly chaotic rotation of chains during the process of sedimentation, what causes the increase of acoustic attenuation. The last one is the decrease of acoustic attenuation to the value as for clean silicone oil. It 
can be supposed that this effect is caused by the sedimentation of particles. The process of sedimentation for MF has not been observed since the mass of nanoparticles or created structures is much smaller than the mass of microparticles used in MR fluids. Very interesting phenomena was the immediate decrease of acoustic attenuation after anisotropy measurements, when the magnetic field was switched off. This means that long time application of the magnetic field to static MR fluids created stable big chains which sediment considerably without the magnetic field.

The anisotropy of acoustic attenuation was measured for three temperatures $\left(15^{\circ} \mathrm{C}, 25^{\circ} \mathrm{C}\right.$ and $\left.35^{\circ} \mathrm{C}\right)$ in constant magnetic field of $200 \mathrm{mT}$ (Fig. 5). As it can be seen from the results, there is the significant effect of the angle between the magnetic field and the wave vector on the acoustic attenuation what is coupled with the process of chains orientation along the direction of magnetic field. Evidently, there are two local maxima at angles $30^{\circ}$ and $75^{\circ}$, as can be observed from the anisotropy measurements result. The first local maximum is dependent on the temperature, while the other is not. It should be noted that the anisotropy measurements of MR fluid of the acoustical properties on $\boldsymbol{B}$ and $\varphi$ are similar to the dependencies obtained when MF has instable structure or subjected before to the magnetic field during some days $[3,26]$. In MF case, sizes of the formed aggregates of particles are relatively close to particles or aggregates which are in MR fluid. Dynamic effects caused by complicated interaction of magnetic field with such structure - large chains or big clusters may initiate a new non-local mechanism of relaxation processes, like the peculiarities of dipole-dipole interaction, magnetostriction and some other effects. These effects explain why two local maxima were observed. The first one is mainly connected with more complex structures (the influence of translational vibrations and rotational degrees of freedom of structures [20,22]) which further decreases with temperature. Number of microparticles involved into structures decrease with the increasing temperature, so the attenuation is smaller. The second maxima could be connected with the above relaxation processes. The acoustic attenuation is also slightly higher for perpendicular orientation as for parallel orientation.
This could be caused by higher possibility of oscillation of the individual particles or clusters in perpendicular orientation to the chain, as in the direction of the chain.

\section{Conclusions}

The methods of acoustic spectroscopy to determine parameters of MR fluid and its structural change in the magnetic field were used. The acoustic attenuation in a frequency range from 3 to $100 \mathrm{MHz}$ and particle size distribution of MR fluids by the ultrasound spectrometer DT-100 were measured. Magnetorheological fluids immediately create stabilized structures chains after the application of an external magnetic field, which are mainly the result of the interaction of microparticles magnetic moments with the field. This phenomenon was confirmed by the jump change of the magnetic field to $200 \mathrm{mT}$ at various temperatures. The slow change on decrease of acoustic attenuation to the similar value as for clean silicone oil after switching the magnetic field off was caused by sedimentation. The study of the anisotropy of acoustic attenuation showed also the importance of the orientation of magnetic field in the MR fluids structure. For better describing dependencies between acoustical and structural parameters of MR fluid, it is necessary to know not only the peculiarities of interaction between microparticles and chains and internal field but also to determine the mechanism of interaction of dispersive phase on the boundary fluid-solid and relaxation processes.

\section{Acknowledgment}

This work was supported by project VEGA $1 / 0510 / 17$ and ITMS: 26210120021, co-funded from EU sources and European Regional Development Fund. This work was supported by the Ministry of Education, Youth and Sports of the Czech Republic - Program NPU I (LO1504).

\section{References}

[1] RODRIGUEZ-LOPEZ, J., SEGURA, L. E., ESPINOSA-FREIJO, F. M.: Ultrasonic Velocity and Amplitude Characterization of Magnetorheological Fluids under Magnetic Fields. Journal of Magnetism and Magnetic Materials, 324, 222-230, 2012.

[2] BOSSIS, G., LACIS, S., MEUNIER, A., VOLKOV, O.: Magnetorheological Fluids. Journal of Magnetism and Magnetic Materials, 252, 224-228, 2002.

[3] BAEV, A. R., KOROBKO, E. V., NOVIKAVA, Z. A.: Acoustical Properties of Magnetorheological Fluids under Applied Magnetic Field. Journal of Intelligent Material Systems and Structures, 26(14), 1913-1919, 2015.

[4] KOROBKO, E. V., NOVIKOVA, Z. A., SERMYAZHKO, E. S., MURASHKEVICH, A. N., ESHENKO, L. S.: Time Stability Studies of Electrorheological Response of Dispersions with Different Types of Charge Carriers. Journal of Intelligent Material Systems and Structures, 26(14), 1782-1788, 2015. 
[5] BARANWAL, D., DESHMUKH, T. S.: MR-Fluid Technology and Its Application - A Review. International Journal of Emerging Technology and Advanced Engineering, 2(12), 563-569, 2012.

[6] DONG, X., MA, N., QI, M., LI, J., GUAN, X., OU, J.: Properties of Magneto-Rheological Fluids Based on Amorphous MicroParticles. Transactions of Nonferrous Metal Society of China, 22, 2979-2983, 2012.

[7] KCIUK, M., TURCZYN, R.: Properties and Application of Magnetorheological Fluids, Journal of Achievements in Materials and Manufacturing Engineering, 18(1-2), 127-130, 2006.

[8] SEDLACIK, M., PAVLINEK, V., LEHOCKY, M., JUNKAR, I., VESEL, A.: Plasma-Enhanced Chemical Vapour Deposition of Octaflourocyclobutane onto Carbonyl Iron Particles. Materiali in Tehnologije, 46(1), 43-46, 2012.

[9] WANG, Z., FANG, H., LIN, Z., ZHOU, L.: Dynamic Simulation Studies of Structural Formation and Transition in ElectroMagneto-Rheological Fluids. International Journal of Modern Physics B, 15(6, 7), 842-850, 2001.

[10] MELlE, S., CALDERON, O. G., RUBIO, M. A., FULlER, G. G.: Microstructure Evolution in Magnetorheological Suspensions Governed by Mason Number. Physical Review E, 68(4), 041501-041511, 2003.

[11] HOCKICKO, P., BURY, P., MUNOZ, F., MUNOZ-SENOVILLA, L.: Investigation of Acoustic and Electrical Properties of a $\mathrm{LiPO}_{3}$ Metaphosphate Glass. Communications - Scientific Letters of the University of Zilina, 16(1), 45-49, 2014.

[12] MUNOZ-SENOVILLA, L., BIRESOVA, J., HOCKICKO, P., MUNOZ, F.: Investigation of the Relationships between Acoustic Attenuation and Ionic Conduction of Metaphosphate Glasses. Journal of Non-crystalline Solids, 440, 26-30, 2016.

[13] KOLTUNOWICZ, T. N., et al.: Study of Dielectric Function of (FeCoZr)(x)(CaF2)((100-x)) Nanocomposites Produced with a Beam of Argon Ions. Journal of Alloys and Compounds, 650, 262-267, 2015.

[14] RAJNAK, M., TIMKO, M., KOPCANSKY, P., PAULOVICOVA, K., TOTHOVA, J., KURIMSKY, J., DOLNIK, B., CIMBALA, R., AVDEEV, M.V., PETRENKO, V.I., FEOKTYSTOV, A.: Structure and Viscosity of a Transformer Oil-Based Ferrofluid under an External Electric Field. Journal of Magnetism and Magnetic Materials, 431, 99-102, 2017.

[15] BRAMANTY, M. A., MOTOZAWA, M., TAKUMA, H., FAIZ, M., SAWADA, T.: Experimental Analysis of Clustering Structures in Magnetic and MR Fluids using Ultrasound. Journal of Physics: Conference Series, 149, 012040, 2009.

[16] BRAMANTY, M. A., MOTOZAWA, M., SAWADA, T.: Ultrasonic Propagation Velocity in Magnetic and Magnetorheological Fluids Due to an External Magnetic Field. Journal of Physics: Condensed Matter, 22, 324102, 2010.

[17] PATEL, J. K., PAREKH, K.: Effect of Carrier and Particle Concentration on Ultrasound Properties of Magnetic Nanofluids. ULTRASONICS, 55, 26-32, 2015.

[18] ODENBACH, S.: Ferrofluids-Magnetically Controlled Suspensions. Colloids and Surfaces A: Physicochemical and Engineering Aspects, 217, 171-178, 2003.

[19] ROZYNEK, Z., et al.: Structuring from Nanoparticles in Oil-Based Ferrofluids. European Physics Journal E, 34(3), 2011.

[20] HORNOWSKI, T., JOZEFCZAK, J., KOLODZIEJCZYK, B., TIMKO, M., SKUMIEL, A., RAJNAK, M.: The Effect of Particle Aggregate Shape on Ultrasonic Anisotropy in Concentrated Magnetic Fluids. Journal of Physics D: Applied Physics, 48(17), 175303, 2015.

[21] KUDELCIK, J., BURY, P., KOPCANSKY, P., TIMKO, M.: Temperature Effect on Anisotropy of Acoustic Attenuation in Magnetic Fluids Based on Transformer Oil. Communications - Scientific Letters of the University of Zilina, 16(1), 33-38, 2014.

[22] SKUMIEL, A.: The Effect of Temperature on the Anisotropy of Ultrasound Attenuation in a Ferrofluid. Journal of Physics D: Applied Physics, 37(22), 3073, 2004.

[23] [Online]. Available: https://www.stavochemie.cz/t1/LZK_TL_Lukosiol.pdf.

[24] Technical Papers of QUANTACHROME: PARTICLE WORD, Edition 3, 2009 [online]. Available: internet: http://www. quantachrome.de/4129-bD1lbg-/de/Home/home.html.

[25] DUKHIN, A. S., GOETZ, P. J.: Characterization of Liquids, Nano- and Microparticulates, and Porous Bodies using Ultrasound. ELSEVIER, New York, p. 503, 2002.

[26] KUDELCIK, J., BURY, P., DRGA, J., KOPCANSKY, P., ZAVISOVA, V., TIMKO, M.: Comparison of Theories of Anisotropy in Transformer Oil-Based Magnetic Fluids. Advances in Electrical and Electronic Engineering, 11(2), 147-155, 2013.

[27] KUDELCIK, J., BURY, P., HARDON, S., SEDLACIK, M., MRLIK, M.: Study of Structural Changes in Magneto-Rheological Fluids by Acoustic Spectroscopy. ELEKTRO 2016, Slovakia, 624-627, 2016. 